\title{
Le futur des paysages suisses: sommes-nous atteints de schizophrénie?
}

\begin{abstract}
Résumé
Le paysage, compris comme l'ensemble des formes visibles de l'espace géographique et des écosystèmes, ainsi que leurs images mentales, peut s'analyser à diverses échelles. L'aménagement du paysage en Suisse révèle un écart considérable entre la valeur accordée au paysage-spectacle et la prise de conscience souvent très limitée des mécanismes menant à sa dénaturation. On observe quatre attitudes principales face au paysage, qui révèlent des symboliques et des systèmes de valeurs distincts, attitudes qui peuvent se définir à travers un paysage typique: la ville à la campagne, le terroir villageois, le décor, le biopaysage. Selon l'emprise que ces diverses attitudes auront sur la société suisse du siècle prochain, les paysages évolueront de manière fort différentes, en matière d'intensité de l'usaige agricole, de dépérissement des forêts ou d'extension des aires construites.
\end{abstract}

\section{Riassunto}

Il paesaggio, definito come l'insieme delle forme visibili dello spazio geografico e degli ecosistemi, ed anche delle loro immagini mentali, può essere analizzato a diverse scale. La pianificazione del paesaggio in Svizzera svela una differenza notevole tra il valore assegnato al paesaggio-spettacolo e la presa di coscienza spesso molto limitata dei meccanismi che conducono alla sua alterazione. Si osservano quattro attitudini che possono essere definite con l'aiuto di un paesaggio tipico: la città in campagna, il paese, la scena, il biopaesaggio. Secondo l'influenza che queste diverse attitudine avranno sulla società svizzera del prossimo secolo, i paesaggi subiranno un'evoluzione molto diversa, per quello che riguarda l'uso agricolo, il deperimento delle foreste o l'estensione delle aree costrutte.

\section{Le paysage}

Le terme de paysage (Landschaft, paesaggio) est un enfant chéri de la géographie, mais elle doit le partager avec beaucoup d'autres disciplines. De plus, c'est un terme du langage courant qui a connu beaucoup de connotations différentes. Il n'est donc pas possible d'imaginer le futur des paysages suisses sans repartir du passé.

Des historiens comme FRANÇOIS WALTER (1990) ont montré que la notion de paysage a été très liée, pour les Suisses, à celle de nature et, également de patrie. La défense de la nature et celle du paysage, très tôt associées, ont donc eu, depuis fort longtemps, une connotation politique et idéologique.

D'autres travaux (MONDADA, PANESE et SÖDERSTRÖM 1992, par exemple) ont souligné, à juste titre, que le terme désignait aussi bien la chose que sa représentation et qu'il y a toute une histoire de l'art pictural du paysage. La représentation des formes du relief, de l'hydrographie, de la faune et de la flore font autant partie de l'analyse géographique que l'étude de ces objets eux-mêmes.

Pour encore mieux brouiller les pistes, l'usage du terme paysage - comme d'ailleurs ceux d'espace ou de géographie - s'est enflé de manière à désigner des entités tout à fait décollées d'une morphologie quelconque du territoire, ainsi le "paysage audiovisuel» ou le "paysage des partis» ou "de la production romanesque»..

Cette approche, aussi pertinente qu'elle soit, néglige toutefois le fait que le paysage est aussi lié au territoire, qu'il est donc espace vécu, indispensable à notre identification aussi bien personnelle que collective et, enfin, espace aménagé.

Il est question ici de paysages produits, construits, transformés, puisque les paysages vierges de toute influence anthropique n'existent plus, sans oublier que, derriere nos manières de les travailler, de les parcourir ou de nous les approprier, nous retrouvons les systemes de valeurs en même temps que quelques-uns des mythes essentiels de toute société humaine.

Dans ce bref essai, le paysage sera entendu comme l'ensemble des formes visibles de l'espace géographique et des écosystèmes en y incluant non seulement les informations dites "objectives» mais aussi les jugements et les images mentales que nos représentations véhiculent à leur propos.

\section{Gestion et aménagement du paysage}

Alors que la gestion du paysage englobe toute action humaine sur l'espace géographique, depuis la plus modeste, telle la cueillette d'une fleur ou d'un fruit jusqu'à la réalisation d'un grand ouvrage, tel un barrage, l'aménagement

Laurent Bridel, Prof., Université de Lausanne, Institut de Géographie, BFSH 2, 1015 Lausanne-Dorigny 
du paysage implique un processus de planification et de programmation tourné vers une modification de la situation de départ donc un degré nettement plus élevé de réflexivité et des mécanismes sociaux plus complexes.

Le niveau auquel se déroule l'aménagement du paysage est étroitement lié à la notion d'échelle et contribue à définir la nature du mécanisme de transformation. Le micro-aménagement est celui du jardinier, de l'horticulteur, du paysan travaillant un champ ou du vacher responsable d'un alpage, donc des opérations pouvant être couvertes d'un coup d'œil, gérées au jour le jour, par un individu. Le projet, même s'il peut se représenter à l'échelle du 1:100e, est d'une autre nature, il ne peut pas prendre forme sans un degré supérieur d'abstraction, de programmation, sans des transactions sociales; il est généralement destiné à durer plus longtemps que le microaménagement. L'ensemble construit, le terroir, la forêt, le site naturel forment une troisième catégorie de niveau qui implique un plus grand nombre d'acteurs, un processus non plus seulement administratif mais nécessairement politique et, généralement, une échelle allant du 1:2000e au 1:25000e. Enfin, il faut introduire un quatrième ni- veau, celui que l'aménagiste ou le géographe vont justement qualifier de paysage, englobant plusieurs unités de base, que l'on définisse celles-ci à l'aide de critères écologiques, fonctionnels, esthétiques ou juridiques. Cette notion, que l'on retrouve notamment dans les inventaires nationaux ou cantonaux de paysages d'intérêt national ou cantonal à protéger (par exemple l'IFP), est aussi à la base de la plupart des discours des géographes sur le paysage (G. GROSJEAN, 1986).

La notion d'aménagement du paysage est d'autant plus difficile à cerner que plusieurs catégories de professionnels et plusieurs lois ou ensembles législatifs traitent de cette matiere.

D'un côté, nous trouvons toutes les lois relatives aux processus de production et aux fonctions de base des espaces non urbains: agriculture, forêt, économie des eaux. Ces domaines concernent avant tout des ingénieurs et techniciens, agronomes, horticulteurs, forestiers en particulier. Les lois de protection de la nature, des monuments et des sites (ou paysages), terrains de prédilection des biologistes, des écologistes mais aussi des historiens de l'art constituent une deuxième catégorie, pas toujours

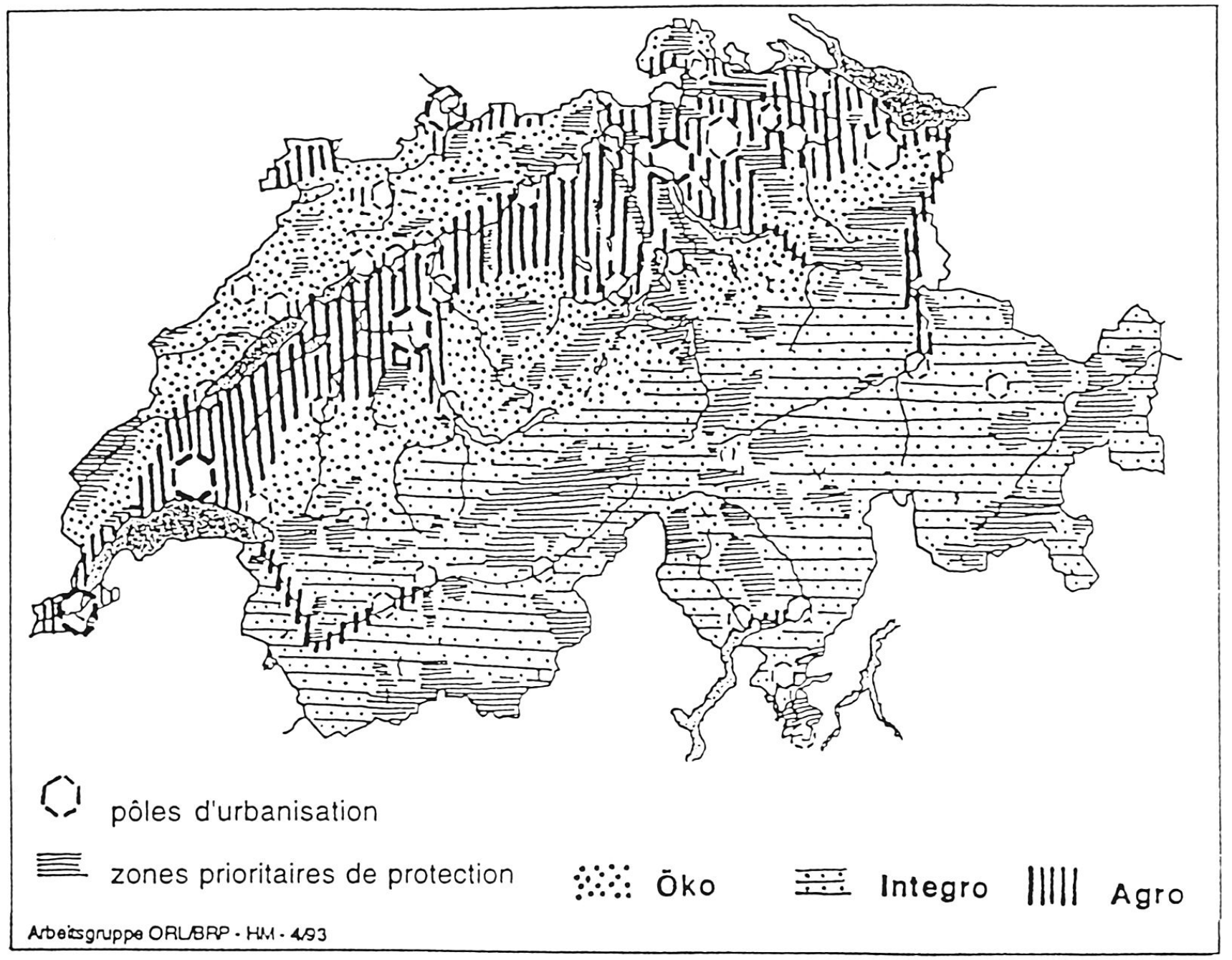

Figure 1 
Tableau 1

\begin{tabular}{|c|c|c|c|c|c|}
\hline $\begin{array}{l}\text { Attitudes face } \\
\text { au paysage }\end{array}$ & $\begin{array}{l}\text { Types } \\
\text { d'exploitation } \\
\text { du paysage }\end{array}$ & $\begin{array}{l}\text { Modes } \\
\text { d'aménagement } \\
\text { du paysage et } \\
\text { de gestion de } \\
\text { l'environnement } \\
\text { construit (EC) }\end{array}$ & $\begin{array}{l}\text { Attitudes } \\
\text { sociales } \\
\text { valorisées }\end{array}$ & $\begin{array}{l}\text { Objets } \\
\text { symboliques }\end{array}$ & $\begin{array}{l}\text { Mythes } \\
\text { et idéologies } \\
\text { sous-jacents }\end{array}$ \\
\hline $\begin{array}{l}1 . \\
\text { La ville à la } \\
\text { campagne }\end{array}$ & $\begin{array}{l}\text { - agglomérations } \\
\text { peu denses, } \\
\text { - habitation } \\
\text { individuelle ou } \\
\text { habitat groupé, } \\
\text { - haute technicité, } \\
\text { - agriculture } \\
\text { intensive, } \\
\text { - forêt mêlée aux } \\
\text { habitations. }\end{array}$ & $\begin{array}{l}\text { aménagement } \\
\text { esthétisant } \\
\text { E.C.: protection } \\
\text { des monuments }\end{array}$ & $\begin{array}{l}\text { individualisme } \\
\text { hédonisme }\end{array}$ & $\begin{array}{l}\text { la maison et } \\
\text { son jardin } \\
\text { le micro-ordina- } \\
\text { teur (domotique) }\end{array}$ & $\begin{array}{l}\text { utopie de } \\
\text { "Broadacre» } \\
\text { libéralisme }\end{array}$ \\
\hline $\begin{array}{l}2 . \\
\text { Le village } \\
\text { et son terroir }\end{array}$ & $\begin{array}{l}\text { - la ville }= \\
\text { ensemble de } \\
\text { quartiers, } \\
\text { - déconcentration } \\
\text { de l'habitat, } \\
\text { - agriculture } \\
\text { traditionnelle ou } \\
\text { friches, } \\
\text { - petites stations } \\
\text { de tourisme, } \\
\text { - forêt jardinée. }\end{array}$ & $\begin{array}{l}\text { aménagement } \\
\text { défensif } \\
\text { (contre } \\
\text { les risques } \\
\text { naturels) } \\
\text { E.C.: protection } \\
\text { des ensembles }\end{array}$ & $\begin{array}{l}\text { défense du } \\
\text { patrimoine } \\
\text { identité collective } \\
\text { (ethnisme) } \\
\text { société } \\
\text { disciplinée }\end{array}$ & $\begin{array}{l}\text { le village avec } \\
\text { l'église au milieu } \\
\text { l'allée d'arbres } \\
\text { le bocage } \\
\text { (Hecken- } \\
\text { landschaft) }\end{array}$ & $\begin{array}{l}\text { la forêt du petit } \\
\text { chaperon rouge: } \\
\text { le méchant loup, } \\
\text { le gentil chasseur } \\
\text { et le charbonnier } \\
\text { romantisme }\end{array}$ \\
\hline $\begin{array}{l}3 . \\
\text { Le décor }\end{array}$ & $\begin{array}{l}\text { - accent mis sur } \\
\text { les espaces } \\
\text { publics et } \\
\text { bâtiments } \\
\text { représentatifs } \\
\text { en ville, } \\
\text { - accent sur } \\
\text { couloirs des } \\
\text { grandes voies } \\
\text { et grandes aires } \\
\text { de loisirs, } \\
\text { - agriculture et } \\
\text { sylviculture } \\
\text { intensives, } \\
\text { - usage maximum } \\
\text { des eaux. }\end{array}$ & $\begin{array}{l}\text { aménagement } \\
\text { productiviste } \\
\text { E.C.: remise } \\
\text { à neuf (à la } \\
\text { japonaise) }\end{array}$ & $\begin{array}{l}\text { l'esprit } \\
\text { d'entreprise } \\
\text { fonctionnel = } \\
\text { beau }\end{array}$ & $\begin{array}{l}\text { le pont } \\
\text { le barrage } \\
\text { le terroir irrigué }\end{array}$ & $\begin{array}{l}\text { le progrès } \\
\text { "papa, je veux } \\
\text { devenir pilote" } \\
\text { prométhée } \\
\text { rationalisme } \\
\text { scientifique }\end{array}$ \\
\hline $\begin{array}{l}4 . \\
\text { Le biopaysage }\end{array}$ & $\begin{array}{l}\text { - villes dans la } \\
\text { verdure, usage } \\
\text { minimal d'énergie } \\
\text { non renouvelable, } \\
\text { - agriculture et } \\
\text { sylviculture } \\
\text { pour maintenir } \\
\text { écosystèmes, } \\
\text { - moins d'usage } \\
\text { de l'eau, surfaces } \\
\text { inondables. }\end{array}$ & $\begin{array}{l}\text { aménagement } \\
\text { réparateur } \\
\text { E.C.: protection } \\
\text { minimale }\end{array}$ & $\begin{array}{l}\text { attention portée } \\
\text { au bio-rythmes } \\
\text { la nature est une } \\
\text { valeur en soi } \\
\text { règles collectives } \\
\text { minimisant les } \\
\text { atteintes aux } \\
\text { écosystèmes }\end{array}$ & $\begin{array}{l}\text { l'étang } \\
\text { la mare } \\
\text { la maison } \\
\text { "biologique" }\end{array}$ & $\begin{array}{l}\text { jardin d'Eden } \\
\text { Tarzan } \\
\text { écologisme }\end{array}$ \\
\hline
\end{tabular}




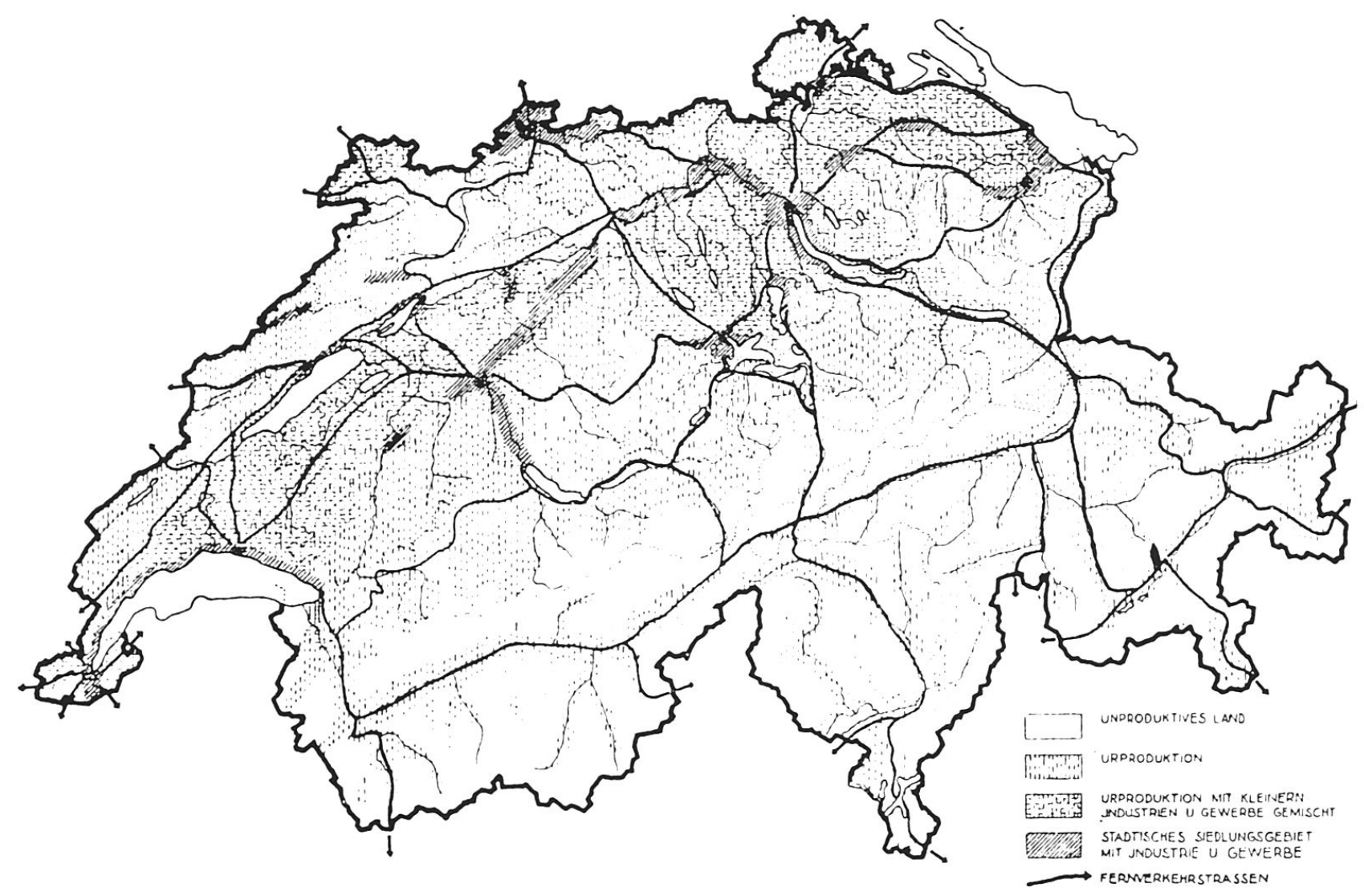

Figure 2 Vorschlag für eine Zoneneinteilung als Grundlage der schweizerischen Landesplanung.

Projet d'une division en zones comme base des divisions principales du territoire suisse.

Source: A. Meili "Allgemeines über Landesplanung" in Die Autostrasse no. 2, 1933, Basel

facile à séparer de celle de la protection de l'environnement qui remet en avant les compétences de l'ingénieur, notamment le chimiste. Enfin, la législation de l'aménagement du territoire s'appuye sur les compétences des aménagistes, des architectes, des ingénieurs, des géographes. Cette multiplicité d'intervention a sans doute contribué à brouiller les enjeux. La primauté des valeurs visuelles, si prégnantes dans l'histoire de nos relations au paysage, continue à survaloriser les actions et les mesures maintenant ou transformant les formes visibles de notre environnement naturel ou construit, alors que les problèmes fondamentaux sont d'un tout autre ordre, économiques, juridiques, chimiques ou micro-physiques dans le sens de mécanismes qui ne sont pas immédiatement perceptibles, soit qu'ils se passent à une échelle échappant à la vue, soit qu'ils se déroulent trop lentement. Par exemple, une notion aussi fondamentale que celle de charge d'un paysage ne peut que très rarement être définie à travers des critères simplement visuels.

Une autre notion classique d'aménagement du paysage, l'intégration, est peut-être en train de subir une métamorphose similaire. Utilisée très souvent dans la pratique de l'urbanisme, elle s'appuie généralement sur des critères de forme (pente du toit, taille des ouvertures en façade par exemple), mais, en matière de paysage, ce sont toujours plus des mécanismes économiques, sociaux ou écologiques qui conditionnent cette adéquation aux conditions prévalant aux alentours de l'objet considéré. Plus pratiquement encore, les tunnels ont pris des connotations fort différentes en une trentaine d'années. Tout d'abord vus avec suspicion - ils sont fort coûteux - ils sont devenus la solution la plus souvent réclamée au cours de ces dernières années, face aux projets ferroviaires ou autoroutiers, par ceux-là mêmes qui apprécient les paysages. Peu à peu, c'est la gêne auditive qui prend le pas sur la gêne (ou l'agrément) visuel et tend à mettre au second rang l'aspect optique du paysage, alors même que les inconvénients du tunnel (gestion des déblais, consommation d'énergie électrique) restent considérables.

Il existe donc une sorte de schizophrénie - comprise comme une psychose menant à une conduite paradoxale et à une perte de contact avec la réalité - dans l'attitude des Suisses face au paysage. Nous cherchons «le visage aimé de la patrie», nous voulons le protéger à grand renfort de mesures protectrices des formes, alors que celles-ci ne sont que la résultante de mécanismes largement invisibles. Il en découle le besoin de se raccrocher à des es- 
paces symboliques, même s'ils ne devaient plus être que des sites d'exception.

Cette tendance est d'autant plus claire qu'il s'agit d'un discours général et de mesures concernant l'échelle nationale. Nous sommes frappés par la similitude existant entre une illustration du document intitulé "Grandes lignes du développement souhaité de l'organisation du territoire" mis en consultation en août 1993 par l'Office fédéral de l'aménagement du territoire (Groupe de travail OFAT/ORL, p. 37 ) et une esquisse d'aménagement national publiée soixante ans plus tôt par ANTON MEILI (1933).

Dans la figure 1, nous trouvons la proposition de traiter le paysage non urbain au moyen de trois ensembles de mesures, appliquées en vertu d'une différenciation spatiale, liée au caractère montagneux et à l'éloignement de la ville. Nous pensons qu'avant de proposer des politiques qui se distinguent avant tout par la localisation du paysage dans l'espace suisse, il convient de se demander quelles sont les valeurs et conceptions implicites qui se partagent les représentations des habitants de ce pays à propos du paysage.

Nous distinguons quatre attitudes fondamentales sommairement définies à travers une grille d'analyse (tableau

1) comportant cinq rubriques:

- les types d'utilisations les plus significatifs,

- les modes d'aménagement du paysage,

- les attitudes sociales les plus valorisées,

- les objets symboliques,

- les mythes sous-jacents.

\section{Quelle mise en valeur du paysage?}

Chacune des quatre attitudes fondamentales face au paysage se retrouve dans l'une ou l'autre des pratiques ou des discours actuels. On peut dire qu'elles s'articulent autour de divers modes de mise en valeur:

La ville à la campagne est un slogan qui a souvent été évoqué, en particulier à propos des cités-jardins (E. HOWARD 1898). Dans cette perspective, la mise en valeur est tout entière tournée vers la satisfaction de la demande de l'habitant, qui souhaite un maximum de commodités, la proximité du lieu de travail et des services courants, ainsi qu'un environnement naturel agréable. Tout ce qui peut faire le confort d'une habitation doit être à disposition grâce aux innovations les plus récentes de la télématique. des multimédias, des moyens de transport rapides et sans bruit. Le paysage doit être joli, à porté de main, un véritable jardin. Chaque objet patrimonial de valeur doit être classé, protégé, mais tout est traité de manière individuelle, avec un minimum de contraintes collectives et un refus de la grande ville avec ses grands nombres et ses grandes densités. Les pollutions sont vaincues par la technique, en chaque lieu d'émission. Le paysage ressemble à celui imaginé par F.L. WRIGHT sous le nom de "Broadacre» (1932-1945). Son symbole est la villa avec son jardin (figure 3 ).

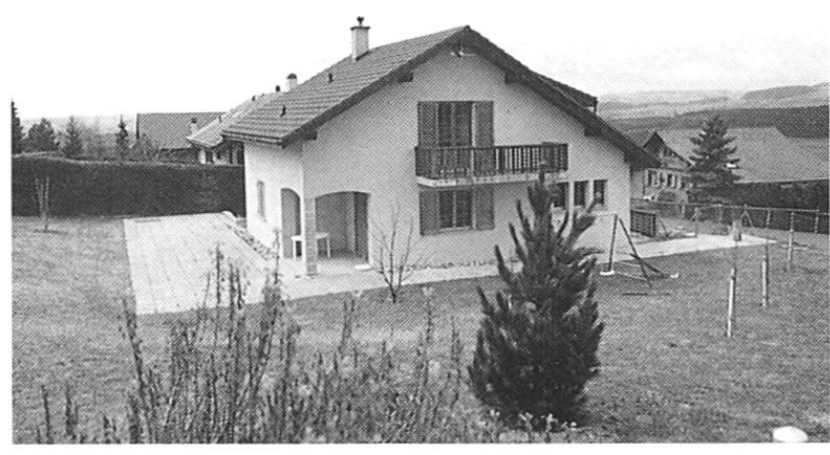

Figure 3

Le village et son terroir est sans doute l'attitude la plus nostalgique, la plus tournée vers un certain passé. Au niveau urbain, les quartiers constituent l'essence de la ville. Le paysage rural est fait de champs comportant encore des arbres et des haies, de vergers agricoles par opposition aux vergers commerciaux, de vignobles peu remaniés, de pâturages boisés, de forêts entrecoupées de clairières et jardinées, à la composition mélangée de feuillus et de coniferes. Les stations de tourisme comme les communes périurbaines s'organisent en villages. C'est une mise en valeur conservatrice, qui s'appuie sur une gestion du patrimoine qui tend à protéger les ensembles plus que les objets individuels.

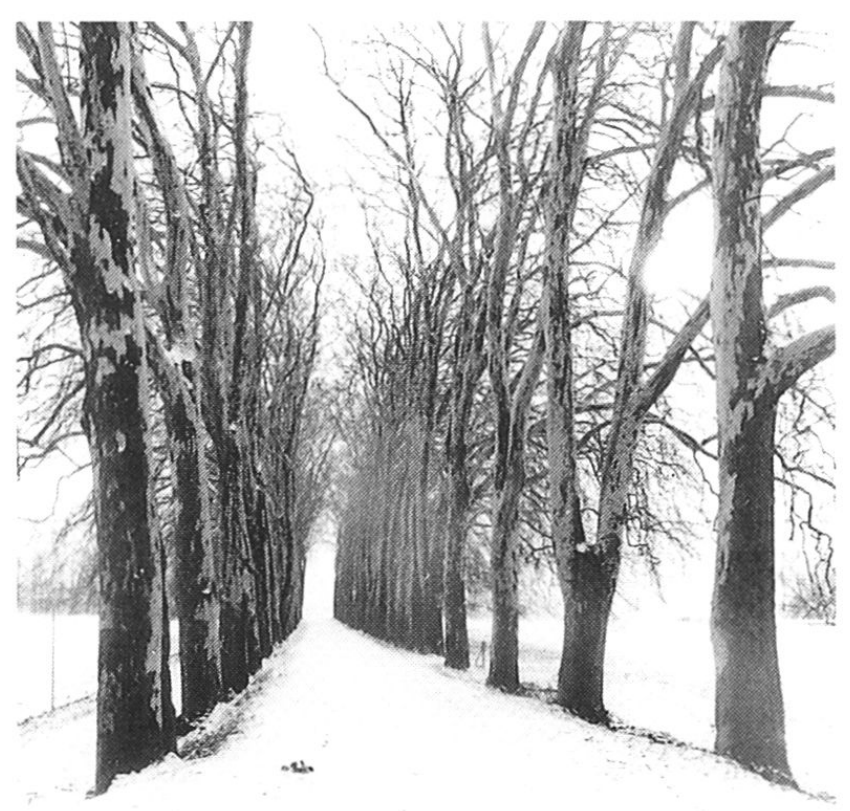

Figure 4 
L'aménagement du paysage sera tourné vers la défense contre les risques naturels, impliquant de strictes disciplines collectives et un minimum de réalisations nouvelles. Plusieurs symboles peuvent illustrer ce système de valeurs teinté de romantisme: l'allée d'arbres, la place du village ou le bocage (figure 4).

L'aménagement du paysage moderniste, productiviste ou progressiste nous paraît caractérisé par la notion de décor. Ce qui importe, c'est ce qui est produit par l'homme, ce qui est rationnel, fonctionnel, efficace. Le paysage doit être ou bien fonctionnel ou bien un décor. A part les étendues agricoles ou forestières qui doivent être aussi homogènes que possible afin de permettre leur mise en valeur économique, dans le sens de l'économie de main-d'œuvre, on distingue la ville des axes les reliants, les lieux de loisirs étant des espèces de villes. En dehors des espaces urbanisés, on aura donc un paysage perçu de manière cinétique. Ce sera un «Kulissenlandschaft», un paysage de couloirs particulièrement illustré par les autoroutes. L'esprit d'entreprise est valorisé, ainsi que la beauté découlant du fonctionnel, des formes simples. Deux symboles surgissent immédiatement à l'esprit, le pont et le barrage (figure 5).

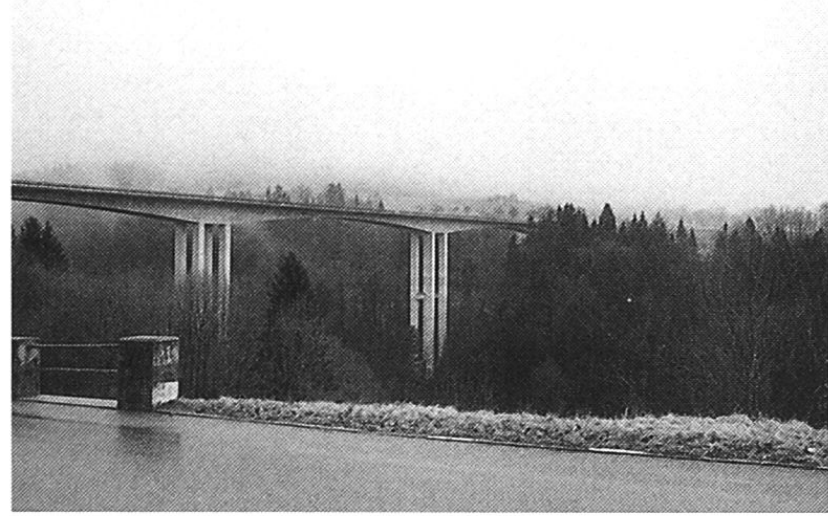

Figure 5

Une gestion des monuments à la japonaise s'accorderait bien à ce type d'attitude; il s'agirait en l'occurrence de reconstruire et de remettre en état les monuments avec des matériaux similaires mais neufs.

Les techniques de simulation visuelle - qu'il s'agisse d'une pièce, d'un bâtiment, d'un arbre, ou encore d'une forêt ou d'un lac de barrage - accompagnent tout naturellement cette conception à la fois technocratique et esthétisante du paysage (S. HEHL-LANGE \& E. LANGE, 1993).

Quant au dernier type analysé, il apparaît comme une antithèse de la mise en valeur, une remise en place ou une mise en défens. Cette attitude, marquée par l'écologisme, veut ramener plus de verdure et de bois dans la ville, plus d'eau dans les aires rurales, moins d'intrants et plus de filtres partout. Étant donné l'état de l'environnement naturel en Suisse, on peut qualifier cet aménagement de réparateur ou correcteur. Cette attitude fait écho à la bio- architecture, aux médecines dites naturelles, à la lutte intégrée en agriculture, à certains accents des mouvements charismatiques. Elle n'implique pas tellement une attitude sociale libertaire que l'appel à des règles collectives strictes minimisant les atteintes à l'environnement. La protection du patrimoine sera donc vue d'un œil critique et neuf et appréciée selon de nouveaux critères; une digue lacustre effondrée pourra paraître plus digne de protection, au titre de niche écologique nouvelle, qu'une chapelle menaçant ruine... Les symboles de cette conception du paysage seraient l'étang et la maison "biologique» en bois et pierre, avec recyclage des eaux, large usage de l'énergie solaire et recours à une arborisation généreuse (figure 6).

\section{Quel paysage en Suisse en 2010?}

Le paysage ne va guère changer de manière dramatique, sauf événement imprévisible. L'essentiel de ses traits de demain sont déjà présents et l'extension de la forêt, sur les friches agricoles, ainsi que l'expansion périurbaine ont déjà fortement marqué le pays.

Il faudrait donc plutôt parler de paysages au pluriel et se demander quels types d'entre eux vont progresser et quels facteurs de changement vont se faire sentir. Nous évoquerons donc brièvement quatre scénarios, construits à partir des attitudes de base (tableau 1) et d'une hypothèse relative à la conjoncture économique (tableau 2).

Étant donné que les scénarios servent surtout de base à une réflexion ou à une discussion, il serait vain de raffiner les hypothèses et de multiplier les facteurs explicatifs, ce qui conduirait à produire une infinité de variantes de toute manière caricaturales. Nous postulons donc que les attitudes décrites ci-dessus peuvent coexister, spécialement deux par deux et que le facteur économique est d'importance majeure pour expliquer l'évolution à venir. Les types de paysage les plus marquants sont d'une part les trois catégories d'aires agricoles ou de végétation non forestière (aires d'agriculture intensive, aires de montagne et aires proches de la nature). Nous entendons par là tous les espaces végétalisés qui ne font partie ni de la forêt de production ou de protection, ni des zones agricoles exploitées avec un certain degré d'intensité, la forêt et les eaux de surface et, d'autre part, les aires urbanisées (centres des villes, banlieues), les petites villes, les localités périurbaines, aires touristiques et rives des lacs.

Les scénarios s'opposent deux à deux: le 1 et le 2 se caractérisent par une situation économique difficile, le 3 et le 4 par une situation de faible chômage; d'autre part, le 1 et le 3 voient les attitudes individualistes, fonctionnalistes et productivistes dominer, alors que, pour le 2 et le 4 ce sont les attitudes patrimonialistes, écologistes et plus collectivistes qui représentent les valeurs dominantes.

Nos présupposés quant aux liens pouvant exister entre ces divers facteurs explicatifs et l'évolution des paysages 


\begin{tabular}{|c|c|c|c|c|}
\hline \multirow[b]{2}{*}{ Facteurs "explicatifs" } & \multicolumn{4}{|c|}{ Scénarios } \\
\hline & $\begin{array}{l}\text { 1. Déprime/ } \\
\text { Déprise }\end{array}$ & $\begin{array}{l}\text { 2. Déprime/ } \\
\text { Reprise }\end{array}$ & $\begin{array}{l}\text { 3. Reprise/ } \\
\text { Bétonnage }\end{array}$ & $\begin{array}{l}\text { 4. Reprise/ } \\
\text { Rajeunissement }\end{array}$ \\
\hline $\begin{array}{l}\text { 1. Attitudes face au paysage } \\
\text { - ville à la campagne } \\
\text { - village } \\
\text { - décor } \\
\text { - biopaysage } \\
\text { 2. Situation économique générale } \\
\text { et ses conséquences sur: } \\
\text { - soutien aux agriculteurs } \\
\text { - prix du bois } \\
\text { - moyens pour conserver } \\
\text { le patrimoine } \\
\text { - activité du secteur } \\
\text { de la construction }\end{array}$ & 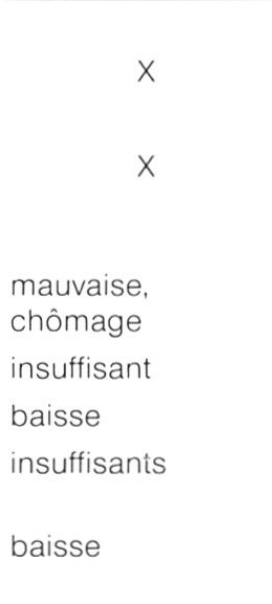 & 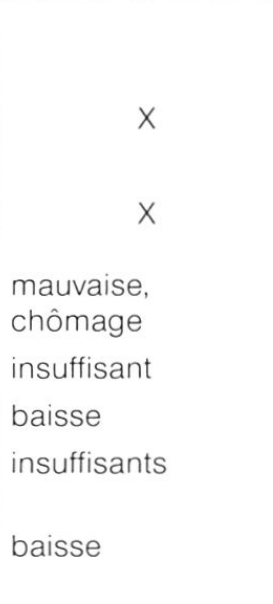 & 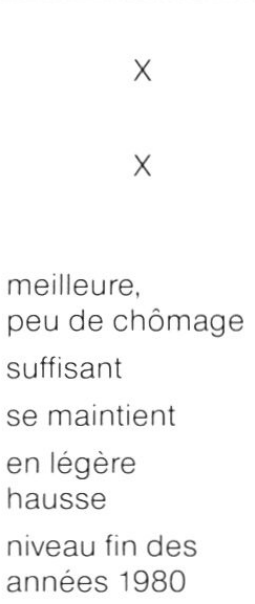 & 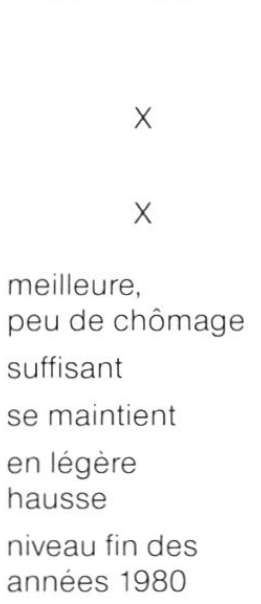 \\
\hline $\begin{array}{l}\text { 3. Effets sur les paysages } \\
\text { Aires agricoles intensives } \\
\text { Agriculture de montagne } \\
\text { Surfaces proches de la nature } \\
\text { Forêt }\end{array}$ & $\begin{array}{l}\text { se réduisent } \\
\text { disparaît } \\
\text { s'étendent sur } \\
\text { friches } \\
\text { s'étend }\end{array}$ & $\begin{array}{l}\text { se réduisent } \\
\text { quelques noyaux } \\
\text { de résistance } \\
\text { s'étendent avec } \\
\text { contrôle } \\
\text { vieillit }\end{array}$ & $\begin{array}{l}\text { se maintiennent } \\
\text { se maintient } \\
\text { s'étendent } \\
\text { anarchiquement } \\
\text { s'améliore }\end{array}$ & $\begin{array}{l}\text { se maintiennent } \\
\text { en bonne santé } \\
\text { s'étend avec } \\
\text { contrôle } \\
\text { s'améliore }\end{array}$ \\
\hline $\begin{array}{l}\text { Centres-villes } \\
\text { Aires sub. et périurbaines }\end{array}$ & $\begin{array}{l}\text { dépérissent } \\
\text { société à deux } \\
\text { vitesses }\end{array}$ & $\begin{array}{l}\text { dépérissent } \\
\text { société à deux } \\
\text { vitesses }\end{array}$ & $\begin{array}{l}\text { se maintiennent } \\
\text { prospèrent }\end{array}$ & $\begin{array}{l}\text { dépérissent } \\
\text { prospèrent }\end{array}$ \\
\hline Eaux & $\begin{array}{l}\text { pas de } \\
\text { changement }\end{array}$ & l'état s'améliore & l'état empire & l'état s'améliore \\
\hline Rives des lacs & sans changement & sans changement & $\begin{array}{l}\text { constructions } \\
\text { accrues }\end{array}$ & $\begin{array}{l}\text { constructions } \\
\text { accrues }\end{array}$ \\
\hline Aires touristiques & se détériorent & $\begin{array}{l}\text { certaines se } \\
\text { maintiennent, } \\
\text { les autres se } \\
\text { détériorent }\end{array}$ & $\begin{array}{l}\text { extension des } \\
\text { constructions }\end{array}$ & $\begin{array}{l}\text { certaines stations } \\
\text { prospèrent, les } \\
\text { autres stagnent }\end{array}$ \\
\hline
\end{tabular}

ressortent du tableau 2. En bref, nous pensons que les aires agricoles intensives se réduiront, dans la pire des éventualités, sans disparaître totalement, alors que l'agriculture de montagne, elle, pourrait presque s'effacer dans le deuxième scénario.

La forêt sera très dépendante de l'état du marché du bois et fort peu des attitudes de la population.

Pour les aires urbaines, nous supposons qu'il y aura de plus en plus une évolution vers une polarisation entre les paysages urbains florissants, verts, accessibles, plus denses et des paysages urbains moins favorisés. Les aires touristiques sont vastes, elles comporteront des paysages qui resteront attrayants pour la clientèle alors qu'ailleurs, du fait du dépeuplement ou, au contraire, d'une banalisation de l'espace envahi par les constructions, les territoires perdront leur potentiel touristique et resteront négligés des autorités.

\section{Références}

ABT, T. (1983): Fortschritt ohne Seelenverlust, Versuch einer ganzheitlichen Schau gesellschaftlicher Probleme am Beispiel des Wandels im ländlichen Raum, Bern.

GROSJEAN, G. (1986): Ästhetische Bewertung ländlicher Räume. Am Beispiel von Grindelwald im Vergleich mit anderen schweizerischen Räumen und in zeitlicher Veränderung. Geographica Bernensia, P 13 Berne.

Groupe de travail Office fédéral de l'aménagement du territoire/Institut ORL EPF Zurich. (1993): Grandes lignes du développement souhaité de l'organisation du territoire, Projet, Berne.

HEHL-LANGE, S. \& LANGE, E. (1993): Digitale und analoge Visualisierung als Entscheidungshilfe in der Planung. Bulletin de l'OFAT No 3-4, p. 24-7. 


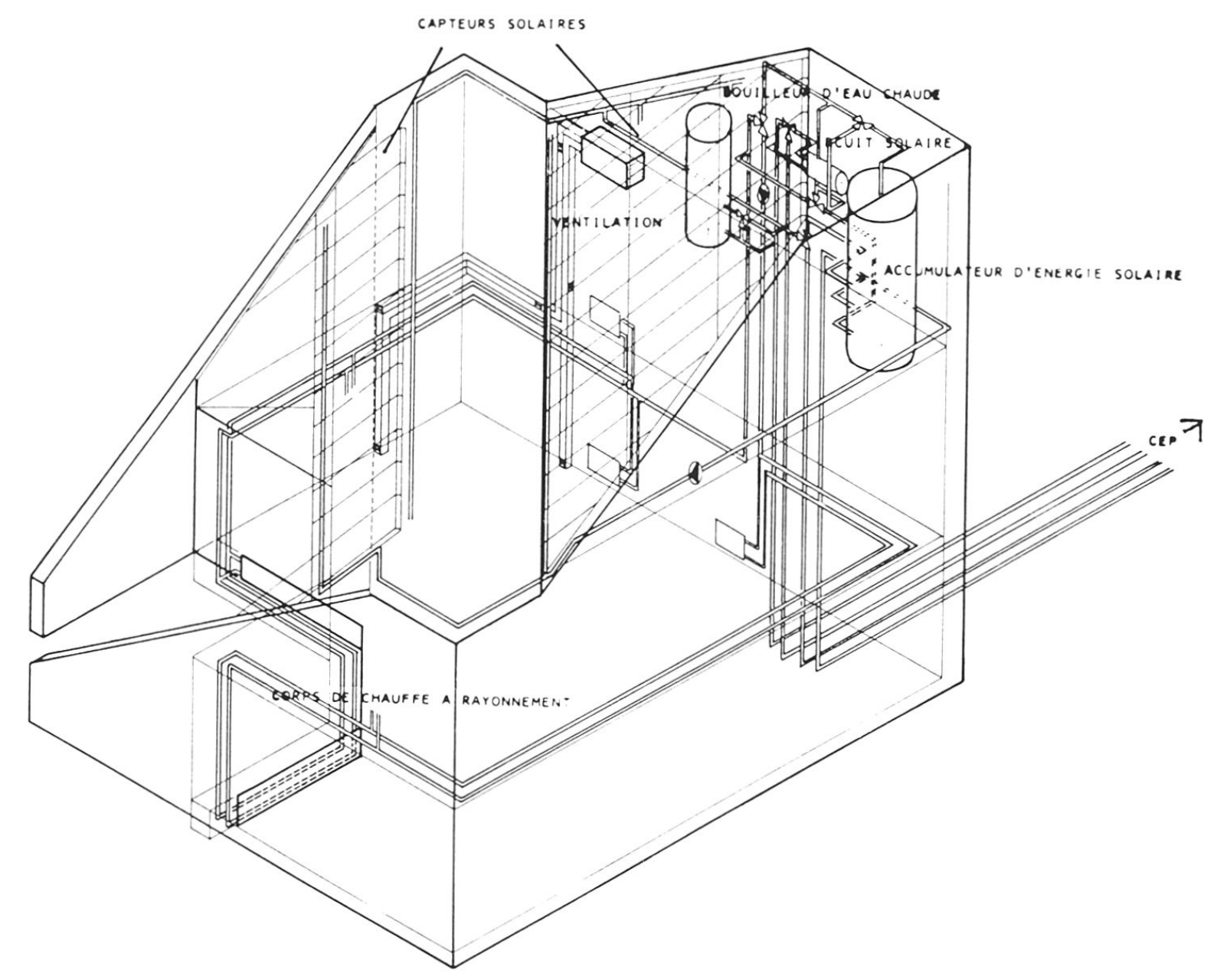

Tiré de Résultats du "Concours pour une maison solaire" organisé par l'IREC et la Ville d'Yverdon, Collection "Etudes urbaines et régionales" dirigée par Michel Bassand et Joseph Csillaghy, Georgi, 1979. Projet HOT de A.J. et M.C. LEMAN architectes à Genève.

Figure 6

HOWARD, E. (1898): Tomorrow: a Peaceful Path to Social Reform; La 2e édition s'intitule: Garden Cities of Tomorrow (nouvelle édition, London, Faber and Faber 1946).

LIZET, B. \& RAVIGNAN, F. de (1987): Comprendre un paysage, guide pratique de recherche, coll. Ecologie et aménagement rural Paris, Institut national de la recherche agronomique.

MEILI, A. (1933): Allgemeines über Landesplanung "Die Autostrasse Nr. 2".

MONDADA, L., PANESE, F. \& SÖDERSTRÖM, O., èd. (1992): Paysage et crise de la lisibilité, Lausanne, Institut de géographie.

ROTACH, M., MAUCH, P., GUELLER, P. (1981): Szenarien künftiger Entwicklungen. Rapport de travail du Programme national de recherche "Problèmes régionaux en Suisse" No 31, Berne

ROUGERIE, G. \& BEROUTCHACHVILI, N. (1991): Géosystèmes et paysages, bilan et méthodes, coll. U Géo. Paris, A. Colin.

WALTER, F. (1990): Les Suisses et l'environnement, une histoire du rapport à la nature du 18 e siècle à nos jours, Collection histoire, Genève, éd. Zoé.

WRIGHT, F. L. (1932): The Disappearing City, New York. WRIGHT, F. L. (1945): When Democracy Builds, Chicago University Press 